\title{
Bafilomycin A1 alleviates depression-like symptoms in chronic unpredictable mild stress rats
}

\author{
ZHIJIAN WANG, SHENGBING LIU, WEIWEI PAN, YANJUN GUO and ZHONGFEI SHEN
}

School of Medicine, Jiaxing University, Jiaxing, Zhejiang 314000, P.R. China

Received February 8, 2018; Accepted July 19, 2018

DOI: $10.3892 / \mathrm{mmr} .2018 .9431$

\begin{abstract}
Major depression is a multifactorial disease. Emerging evidence has suggested that autophagy is involved in the pathological process of depressive disorders. Bafilomycin A1 (Baf A1), is an inhibitor of vacuolar $\mathrm{H}^{+}$-ATPase that is frequently used at high concentrations to block late-phase autophagy. However, whether Baf A1 has antidepressant effects remains to be elucidated. The current study aimed to evaluate the antidepressant effects of Baf A1 in rats with chronic unpredictable mild stress (CUMS) and its potential mechanism. The CUMS animal model was established. The sucrose preference test, open-field test (OFT) and forced swim test (FST) were applied to evaluate the depressive behavior. Synaptic plasticity-associated proteins synaptophysin and postsynaptic density protein 95 were measured by western blotting and immunofluorescence. Apoptosis- and autophagy-associated proteins in addition to pro-inflammatory cytokines, including interleukin- $1 \beta$ and tumor necrosis factor- $\alpha$, were detected by western blotting, reverse transcription-quantitative polymerase chain reaction or ELISA. A 4-week treatment period with Baf A1 markedly ameliorated CUMS-induced behavioral abnormalities, including increasing sucrose intake, improving locomotor activity in the OFT, and decreasing immobility time in the FST. In addition, treatment with Baf A1 restored the dysregulation of synaptic plasticity and inhibited neuroinflammation in rats exposed to CUMS. Furthermore, Baf A1 decreased the levels of apoptosis- and autophagy-associated proteins induced by CUMS. The present study demonstrated that Bafilomycin A1 resulted in antidepressant effects in rats, which may be mediated by the reversal of apoptosis, autophagy and neuroinflammation in the hippocampus.
\end{abstract}

Correspondence to: Dr Zhongfei Shen, School of Medicine, Jiaxing University, 118 Jiahang Road, Jiaxing, Zhejiang 314000, P.R. China

E-mail: zhongfeishen0018@163.com

Key words: bafilomycin A1, depression, autophagy, apoptosis, inflammation

\section{Introduction}

Major depressive disorder (MDD) is a severe psychiatric disease, characterized by anorexia, pessimism and a series of depressive-like behaviors. The World Health Organization reports that depression may become the 2 nd most prevalent disease worldwide by 2020 (1,2). However, the exact mechanism underlying depression remains largely unknown. The majority of current antidepressants are based on monoamine neurochemical alterations, including tricyclic antidepressants, and selective serotonin and noradrenalin reuptake inhibitors (3). Numerous patients suffering from MDD are resistant to currently available antidepressants, experiencing a long delay in activity and adverse effects (4). Consequently, the development of novel antidepressant drugs is a primary research concern.

Numerous studies have demonstrated that the pathophysiology of depression involves complex signaling networks, including abnormal cytokine secretion, monoamine deficiency, and disturbed neurogenesis and neuroplasticity (5). In addition, it has been proposed that dysfunctional synaptic plasticity is a basis of the etiology of depression $(6,7)$. Furthermore, postmortem brain tissues from patients with MDD display increased levels of apoptosis-associated factors (8). Recent studies have indicated that neuronal autophagy signaling pathways are also involved in the pathophysiology of $\operatorname{MDD}(9,10)$. Dysregulation of autophagy may result in a cellular 'traffic jam' during neuronal development and synaptic plasticity, leading to neurodevelopmental disorders (9). Dysregulation of the autophagy pathway in neurons may result in depression (10). Certain antidepressants or molecules alleviate the symptoms of depression by regulating apoptosis or autophagy (10).

Bafilomycin A1 (Baf A1), a macrolide antibiotic isolated from Streptomyces species, is an inhibitor of vacuolar $\mathrm{H}^{+}$-ATPase (11). It has been reported that Baf A1 has potent bioactivity, including inhibition of cell growth, differentiation and apoptosis, and anti-inflammatory and anti-tumor properties $(12,13)$. At high doses (0.1-1 mM) Baf A1 has frequently been used as an inhibitor to block the fusion between autophagosomes and lysosomes, or to inhibit lysosomal activity, a critical step in late-stage autophagy $(12,14)$. Previous research has reported that Baf A1 may improve cell survival in PC12 cells and cerebral ischemia-reperfusion injury in rats by regulating autophagy and the apoptosis pathway (15), suggesting it may serve an important role in diseases of the nervous system. 
However, the antidepressant effects of Baf A1 in chronic unpredictable mild stress (CUMS) depression in rats have not been elucidated.

In the present study, the antidepressant effects of Baf A1 were investigated in a CUMS-induced depressive rat model. The present results revealed that Baf A1 significantly alleviated depressive-like behaviors, which may be mediated by anti-inflammatory effects, the regulation of autophagy and the apoptosis pathway.

\section{Materials and methods}

Animals and drug treatments. A total of 105 Male Sprague Dawley rats (15 weeks old, 180-200 g) were purchased from the Experimental Animal Center of Zhejiang province (Zhejiang, China). Animals were single-housed under controlled temperature and relative humidity $\left(25 \pm 2^{\circ} \mathrm{C}\right.$ and $55 \pm 5 \%$, respectively) and a 12-h light/dark cycle (lights on from $7 \mathrm{am}$ to $7 \mathrm{pm}$ ). Prior to the experiment, the animals were allowed 1 week to adapt to the new environment. Rats were randomly divided into the following groups following three sessions of sucrose training: Control, CUMS, CUMS + fluoxetine $(10 \mathrm{mg} / \mathrm{kg}$ fluoxetine; Sigma-Aldrich; Merck KGaA, Darmstadt, Germany), and CUMS + Baf A1 (10, 25, 50 or $100 \mathrm{mg} / \mathrm{kg}$; Sigma-Aldrich) ( $\mathrm{n}=10$ rats/group). All drugs were dissolved in $0.9 \%$ saline containing $0.5 \%$ carboxymethyl cellulose. After 6 weeks of CUMS exposure, all drugs were orally administered once daily for a further 4 weeks in each group. Following this, behavioral tests were performed and animals were sacrificed for further biochemical determinations. All animal experiments were approved by the Ethics Committee of the Medical College of Jiaxing University (Jiaxing, China). All procedures adhered to the National Institutes of Health Guide for the Care and Use of Laboratory Animals (16).

CUMS procedure. The stress procedure was performed as stipulated in a previous protocol (1). The stressors included swimming in ice water $\left(4^{\circ} \mathrm{C} ; 5 \mathrm{~min}\right)$, a reversed light/dark cycle $(24 \mathrm{~h})$, damp bedding $(200 \mathrm{ml}$ sterile water added to the cage; $12 \mathrm{~h}$ ), noise (100 dB; $5 \mathrm{~min})$, oscillation (2 min), tail pinch ( $1 \mathrm{~cm}$ from the top of the tail; $2 \mathrm{~min}$ ), water and food deprivation $(24 \mathrm{~h})$, cage tilt $\left(45^{\circ} ; 12 \mathrm{~h}\right)$ and foot shock (36 V; duration $10 \mathrm{sec}$, intershock interval $30 \mathrm{sec}, 15$ shocks). Each rat received two or three random stressors daily.

Body weight measurement and sucrose preference test (SPT). Body weight was measured at 9:00 a.m. on the same day each week. during the experimental period. Rats were allowed to adapt to $1 \%$ sucrose solution for $24 \mathrm{~h}$, then deprived of food and water for $24 \mathrm{~h}$ prior to the SPT. Each animal was allowed to imbibe two drinking bottles of $1 \%$ sucrose water or tap water for $12 \mathrm{~h}$. In order to avoid position preference, the positions of the drinking bottles were switched halfway through the test. Finally, the weights of sucrose solution and water consumed were recorded accordingly. The preference of sucrose consumption was defined as the percentage of consumed sucrose water out of the total amount of liquid drunk during the 12-h test. The sucrose preference value was calculated using the following formula: Sucrose preference $(\%)=$ sucrose solution intake $(\mathrm{g}) /[$ sucrose solution intake $(\mathrm{g})+$ water intake $(\mathrm{g})]$ x100. During the experimental period, the SPT was carried out at the end of each week in the same way.

Open-field test (OFT). Rats were placed in an empty square box (40 cm long x60 cm wide x50 cm high) made of compressed wood to evaluate locomotor activity and spontaneous exploration in a novel environment. The apparatus was divided into 12 equal squares and the number of crossings were recorded. OFT was performed as previously described (17). Rats were placed individually into the center of the apparatus and tracked for 5 min with a video camera. The total distance within the last 4 min was recorded. The apparatus was cleaned using a detergent and subsequently dried between each rat test. The data were collected and analyzed using the Open Field Scan system (TopScan Realtime Option Version 2.0; CSI-OF; Clever Sys Inc., Reston, VA, USA).

Forced swim test (FST). The FST was performed as previously described (18). Rats were placed in a glass cylinder $(46 \mathrm{~cm}$ height; $30 \mathrm{~cm}$ diameter; filled with $25 \pm 1^{\circ} \mathrm{C}$ water to a depth of $30 \mathrm{~cm}$ ) for $6 \mathrm{~min}$. During the test, the immobility time was defined as the time for which the rat floated in the water without struggling and only made movements necessary to keep its head above the water. The duration of immobility during the last $5 \mathrm{~min}$ of the test was recorded by a video camera. The data were recorded and analyzed using Tail Suspension Scan (Tail Suspension Scan ${ }^{\mathrm{TM}}$ Version 2.0; CSI-FSW, Clever Sys Inc.).

Western blotting. At the end of the behavioral tests, the rats were sacrificed and the brain region of the whole hippocampus was dissected on a cold plate and immediately frozen in liquid nitrogen. The tissue samples were stored at $-80^{\circ} \mathrm{C}$ until assay. A Cytoplasmic Protein Extraction kit (Beyotime Institute of Biotechnology) was used to extract proteins from hippocampal tissues. The protein concentration was quantified using a bicinchoninic acid protein assay kit (Pierce; Thermo Fisher Scientific, Inc.). The samples $(30 \mu \mathrm{g})$ were separated by 10 or $15 \%$ SDS-PAGE, and electro-transferred to polyvinylidene difluoride membranes (EMD Millipore, Billerica, MA, USA). Membranes were blocked in 5\% skimmed milk at room temperature for $2 \mathrm{~h}$, and incubated with primary antibodies against: Beclin 1 (1:1,000; cat. no. 3738; Cell Signaling Technology, Inc., Danvers, MA, USA), apoptosis regulator Bcl-2 (Bcl-2; 1:1,000; cat. no. 15071; Cell Signaling Technology, Inc.), cleaved caspase-3 (C-Cas-3; 1:1,000; cat. no. 9661; Cell Signaling Technology, Inc.), synaptophysin (SYP; 1:200; cat. no. sc-136271; Santa Cruz Biotechnology, Inc., Dallas, TX, USA), postsynaptic density protein 95 (PSD 95; 1:200; cat. no. sc-71936; Santa Cruz Biotechnology, Inc.), microtubule-associated proteins $1 \mathrm{~A} / 1 \mathrm{~B}$ light chain 3 (LC3; 1:2,000; cat. no. ab48394; Abcam, Cambridge, MA, USA) and $\beta$-actin (1:2,000; cat. no. ab8226; Abcam) overnight at $4^{\circ} \mathrm{C}$. Membranes were incubated with horseradish peroxidase-conjugated anti-rabbit or anti-mouse immunoglobulin (Ig)G secondary antibodies (1:2,000; cat. nos. BA1054 or BA1050; Boster Biological Technology, Pleasanton, CA, USA) at room temperature for $1 \mathrm{~h}$ and enhanced chemiluminescence reagents (Immobilon Western, EMD Millipore) as recommended by the manufacturer. The quantitation analysis was performed using Image $\mathbf{J}$ software (version 4.0; National 

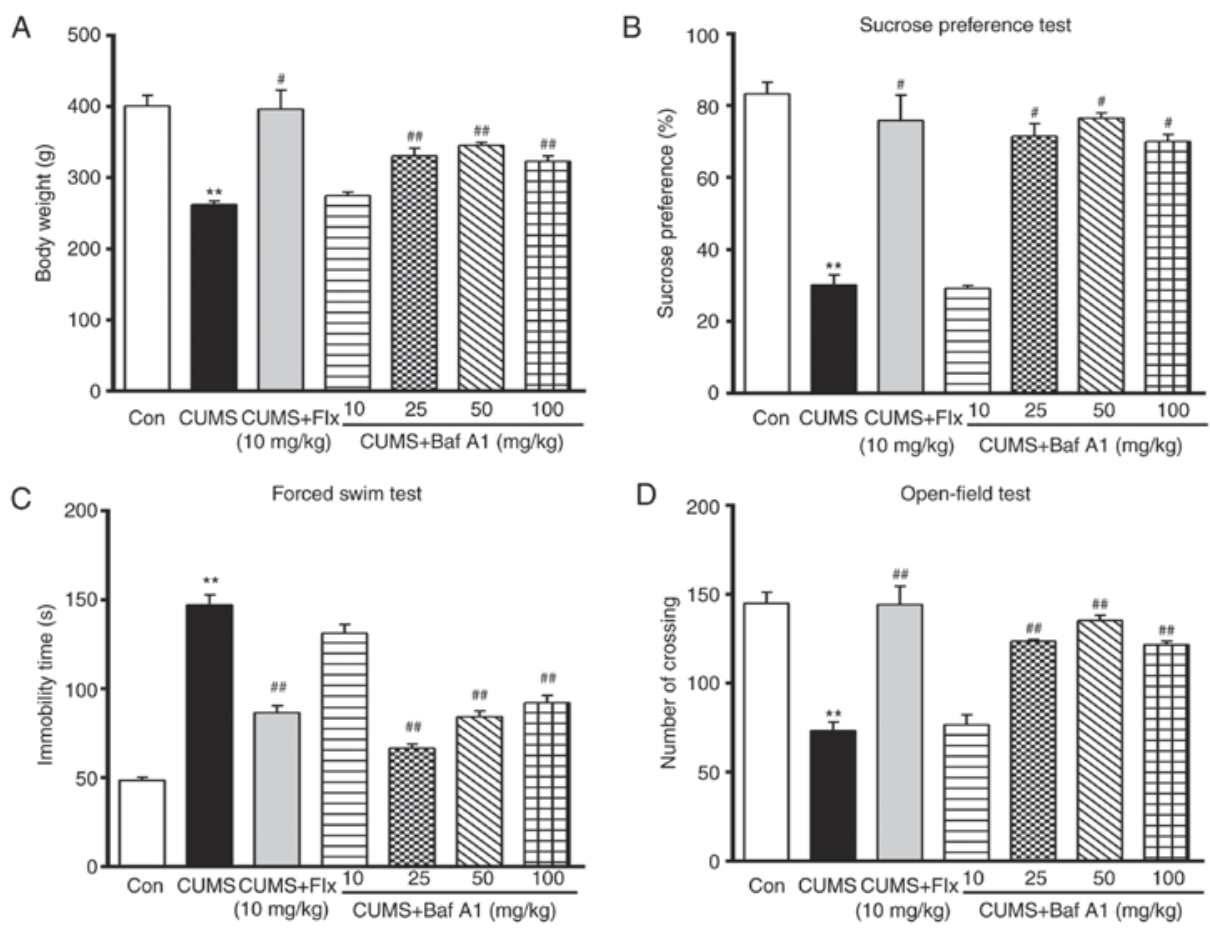

Figure 1. Effects of Baf A1 on CUMS-induced depressive-like behaviors. Effects of Baf A1 (10,25, 50 and $100 \mathrm{mg} / \mathrm{kg}$ ) or Flx (10 mg/kg) on (A) CUMS-induced rat body weight, (B) sucrose preference, $(\mathrm{C})$ immobility time in the forced swim test, and (D) the total distance in the open field test. Data are expressed as the mean \pm standard error of the mean; $\mathrm{n}=10$ per group. ${ }^{* *} \mathrm{P}<0.01$ vs. Con; ${ }^{*} \mathrm{P}<0.05,{ }^{* \# /} \mathrm{P}<0.01$ vs. CUMS. CUMS, chronic unpredictable mild stress; Con, control; Flx, fluoxetine; Baf A1, Bafilomycin A1.

Institutes of Health, Bethesda, MD, USA), and immunoreactivity was normalized to the $\beta$-actin loading control for each protein.

Reverse transcription-quantitative polymerase chain reaction (RT-qPCR). Total RNA was isolated using TRIzol ${ }^{\circledR}$ reagent (Thermo Fisher Scientific, Inc.) from hippocampal tissues and 2-3 $\mu \mathrm{g}$ total RNA was used to synthesize first-strand cDNA using SuperScript II reverse transcriptase kit (Invitrogen; Thermo Fisher Scientific, Inc.), following the manufacturer's protocol. qPCR reactions were performed using $\mathrm{SYBR}^{\circledR}$ Green Realtime Master Mix and ABI PRISM ${ }^{\circledR} 7700$ (Applied Biosystems; Thermo Fisher Scientific, Inc.). The cycling conditions were: Denaturation at $95^{\circ} \mathrm{C}$ for $30 \mathrm{sec}$, followed by 40 cycles of DNA synthesis at $95^{\circ} \mathrm{C}$ for $5 \mathrm{sec}$ and $60^{\circ} \mathrm{C}$ for $34 \mathrm{sec}$. The primers used were: SYP sense, $5^{\prime}$-CATCTTCGC CTTTGCTACG-3'; SYP antisense, 5'-CACTGAGGTGTT GAGTCCTGA-3'; PSD95 sense, 5'-ACAACCAAGAAATAC CGC-3'; PSD95 antisense, 5'-ATACTCCATCTCCCCCTC-3'; $\beta$-actin sense, 5'-CCAGATCATGTTTGAGACC-3'; $\beta$-actin antisense, 5'-ATGTCACGCACGATTTCCC-3'. $\beta$-actin was used as an internal standard. All results were calculated using the $2^{-\Delta \Delta \mathrm{Cq}}$ method (19).

Immunofluorescence. At the end of the behavioral tests, the rats were sacrificed, and the brains were removed and fixed in $4 \%$ paraformaldehyde for $24 \mathrm{~h}$ at $4^{\circ} \mathrm{C}$. For immunofluorescence, all procedures were performed as previously described (20). Sections of the brain at a thickness of $30 \mu \mathrm{m}$ were prepared using standard protocols and stained with primary anti-PSD95 antibody (1:50; cat. no. sc-71936; Santa Cruz Biotechnology, Inc.) overnight at $4^{\circ} \mathrm{C}$, followed by Alexa Fluor 488 goat anti-mouse IgG (1:300; cat. no. B40912; Invitrogen; Thermo Fisher Scientific, Inc.) for $1 \mathrm{~h}$ at $37^{\circ} \mathrm{C}$ and then stained with propidium iodide (PI, $50 \mu \mathrm{g} / \mathrm{ml}$ ) in the dark for $30 \mathrm{~min}$ and observed. Images were captured using a confocal microscope system at x200 magnification (LSM510; Carl Zeiss AG, Oberkochen, Germany).

ELISA. Following the behavioral tests, the rats were sacrificed, and the brains were immediately removed. The hippocampi were dissected and homogenized, then centrifuged at $1,000 \mathrm{x} \mathrm{g}$ for $20 \mathrm{~min}$ at $4^{\circ} \mathrm{C}$. The levels of interleukin (IL) $1 \beta$ (cat. no. RLB00) and tumor necrosis factor (TNF)- $\alpha$ (cat. no. RTA00) were measured using ELISA kits (R\&D Systems, Minneapolis, MN, USA), according to the manufacturer's protocols.

Statistical analysis. Statistical analyses were performed using GraphPad Prism 5.0 Software (version 2.0; GraphPad Software, Inc., La Jolla, CA, USA). Significant differences were analyzed by one-way analysis of variance, followed by Tukey's post hoc test. The data are presented as the mean \pm standard error of the mean of three independent experiments. $\mathrm{P}<0.05$ was considered to indicate a statistically significant difference.

\section{Results}

Baf Al improves depressive-like behaviors in CUMS rats. As presented in Fig. 1A, CUMS rats exhibited decreased body weights compared with control group rats. In addition, CUMS rats exhibited lower sucrose preference, longer durations of forced swim immobility and a reduced crossing time in the OFT when compared with the control group (Fig. 1B-D). A 

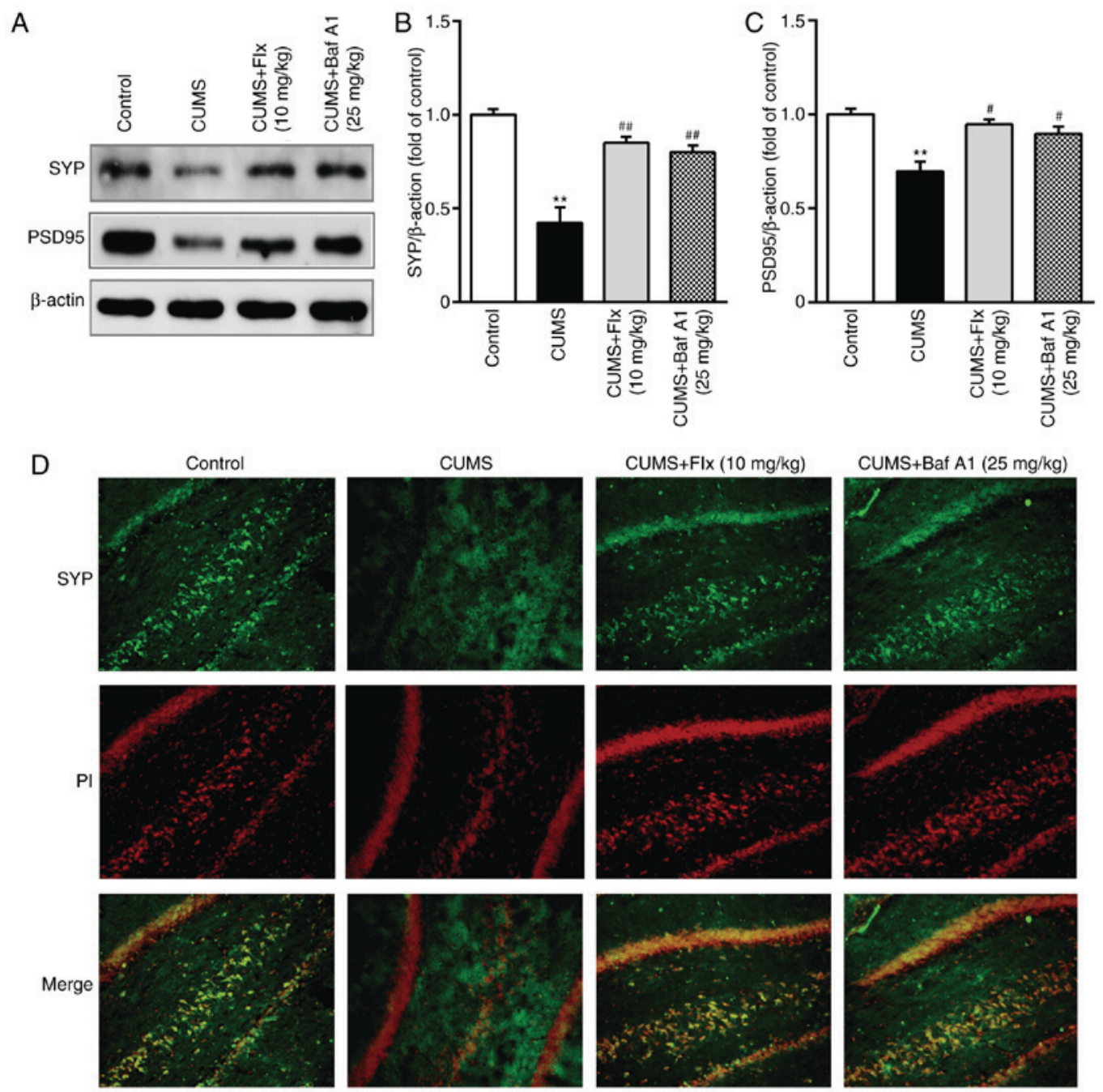

Figure 2. Effects of Baf A1 (25 mg/kg) or Flx (10 mg/kg) on CUMS-induced rat synaptic plasticity-associated proteins SYP and PSD 95. (A) Western blotting results and quantitative analysis of the expression levels of (B) SYP and (C) PSD 95 are presented. $n=10$. (D) Immunoreactivity to SYP (marked in green) in the hippocampus. Magnification, $\mathrm{x} 200 . \mathrm{n}=5$. Data are expressed as the mean \pm standard error of the mean. ${ }^{* *} \mathrm{P}<0.01$ vs. Con; ${ }^{\#} \mathrm{P}<0.05$, ${ }^{\# \#} \mathrm{P}<0.01 \mathrm{vs}$. CUMS. SYP, synaptophysin; PSD 95, postsynaptic density protein 95; CUMS, chronic unpredictable mild stress; Flx, fluoxetine; Baf A1, Bafilomycin A1; PI, propidium iodide.

4-week treatment with Baf A1 $(25,50$ and $100 \mathrm{mg} / \mathrm{kg})$ and fluoxetine $(10 \mathrm{mg} / \mathrm{kg})$ significantly increased the body weights and percentages of sucrose preference, decreased the immobility time in the FST and increased the crossing time in the OFT compared with the CUMS group (Fig. 1A-D). However, there were no statistically significant differences between the CUMS group and the Baf A1 (10 mg/kg) group. The results suggested that Baf A1 exerted antidepressant effects in CUMS rats. However, there was almost no difference between the Baf A1 25, 50 and $100 \mathrm{mg} / \mathrm{kg}$ groups, thus the dose of Baf A1 used in the subsequent experiments was $25 \mathrm{mg} / \mathrm{kg}$.

Baf Al increases synaptic protein expression in CUMS rats. As presented in Fig. 2A-C, the expression levels of SYP and PSD 95 were significantly decreased in the hippocampi of CUMS rats compared with the control group. The administration of Baf A1 $(25 \mathrm{mg} / \mathrm{kg})$ and fluoxetine $(10 \mathrm{mg} / \mathrm{kg})$ for 4 weeks significantly increased the expression levels of SYP and PSD 95 in the hippocampus compared with the CUMS group. Subsequently, the present study also detected the immunoreactivity of SYP in the hippocampi of rats. The immunofluorescence results demonstrated that treatment with Baf A1 $(25 \mathrm{mg} / \mathrm{kg})$ and fluoxetine $(10 \mathrm{mg} / \mathrm{kg})$ markedly increased the fluorescence intensity of SYP compared with the CUMS group (Fig. 2D).

Baf Al ameliorates the inflammatory response in the hippocampi of CUMS rats. Inflammation is closely associated with the development of depressive symptoms, and the anti-inflammatory effects of Baf A1 in CUMS rats were further examined. As demonstrated in Fig. 3, the levels of pro-inflammatory cytokines IL-1 $\beta$ and TNF- $\alpha$ were significantly increased in the hippocampi of CUMS rats compared with the control group. However, this increase was significantly suppressed by treatment with Baf A1 $(25 \mathrm{mg} / \mathrm{kg})$ and fluoxetine $(10 \mathrm{mg} / \mathrm{kg})$ for 4 weeks, suggesting that Baf A1 may have the ability to ameliorate CUMS-induced increases in the levels of IL-1 $\beta$ and TNF- $\alpha$ in the hippocampus.

Baf A1 inhibits hippocampal cell apoptosis in CUMS rats. The expression levels of the apoptosis-associated proteins Bcl-2 and C-Cas-3 in the hippocampi of CUMS rats were assessed 
A

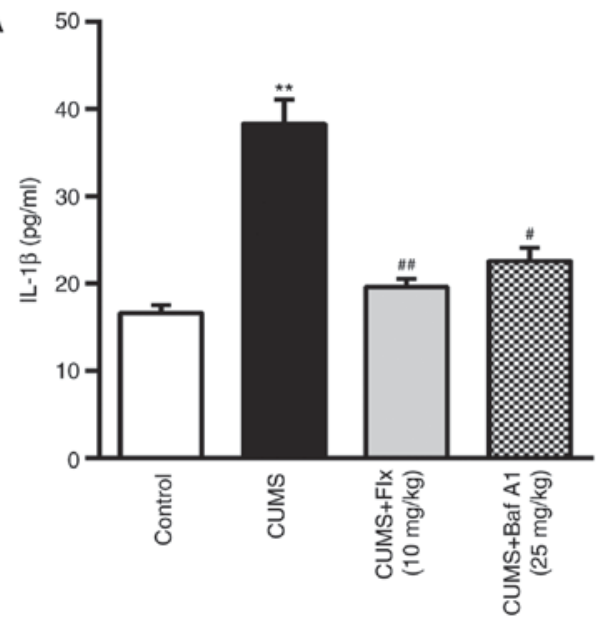

B

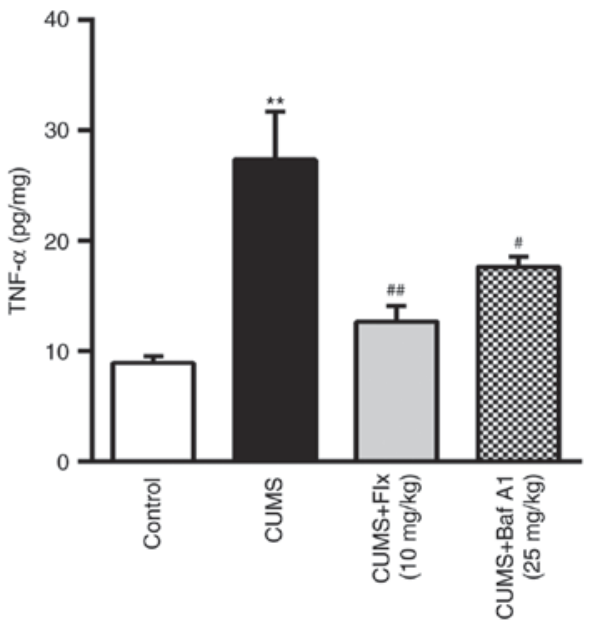

Figure 3. Effects of Baf A1 (25 mg/kg) or Flx (10 mg/kg) on CUMS-induced hippocampal pro-inflammatory cytokines. (A) IL-1 $\beta$ and (B) TNF- $\alpha$ levels were determined by ELISA. Data are expressed as the mean \pm standard error of the mean. $\mathrm{n}=10$ per group. ${ }^{* * *} \mathrm{P}<0.01 \mathrm{vs}$. con; ${ }^{*} \mathrm{P}<0.05$, ${ }^{\# \#} \mathrm{P}<0.01$ vs. CUMS. CUMS, chronic unpredictable mild stress; Flx, fluoxetine; Baf A1, Bafilomycin A1; IL-1 $\beta$, interleukin-1 $\beta$; TNF- $\alpha$, tumor necrosis factor- $\alpha$.
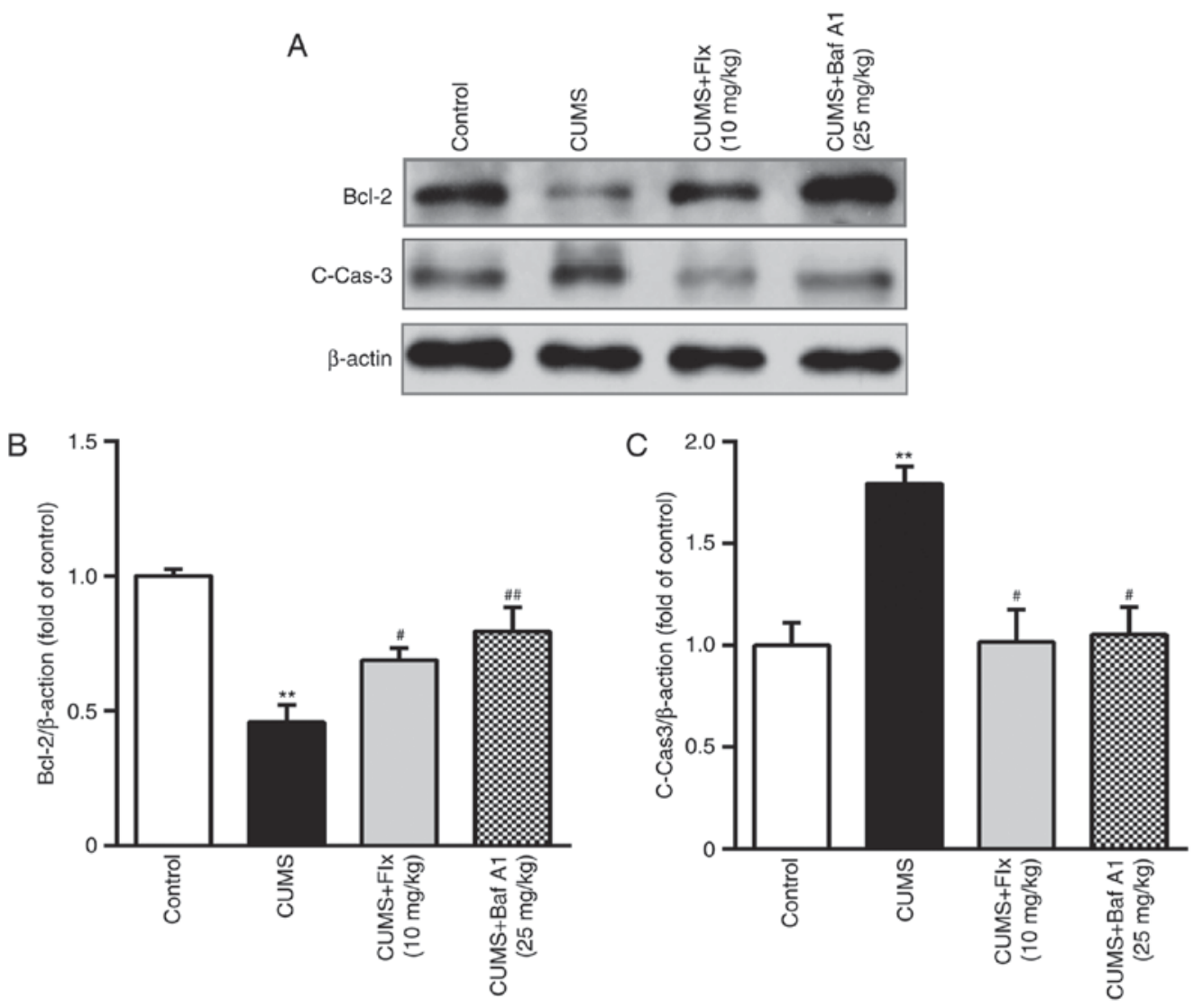

Figure 4. Effects of Baf A1 (25 mg/kg) or Flx (10 mg/kg) on CUMS-induced rat apoptosis-associated proteins Bcl-2 and C-Cas-3. (A) Western blotting results; quantitative analysis of (B) Bcl-2 and (C) $\mathrm{C}$-Cas-3 expression. Data are expressed as the mean \pm standard error of the mean. $\mathrm{n}=10$ per group. ${ }^{* *} \mathrm{P}<0.01$ vs. con; ${ }^{\#} \mathrm{P}<0.05,{ }^{\# \#} \mathrm{P}<0.01$ vs. CUMS. C-Cas-3, cleaved caspase-3; CUMS, chronic unpredictable mild stress; Flx, fluoxetine; Baf A1, Bafilomycin A1; Bcl-2, apoptosis regulator Bcl-2.

by western blotting. As presented in Fig. 4, the expression of Bcl-2 was significantly decreased in the hippocampi of the CUMS group compared with the control group. The expression levels of C-Cas-3 in the hippocampi were significantly increased in the CUMS group compared with the control group. Administration of Baf A1 $(25 \mathrm{mg} / \mathrm{kg})$ and fluoxetine $(10 \mathrm{mg} / \mathrm{kg})$ for 4 weeks significantly increased the expression of Bcl-2 and decreased the expression of C-Cas-3 compared with the CUMS group. These results indicated that Baf A1 inhibited hippocampal cell apoptosis in CUMS rats.

Bafilomycin A1 inhibits autophagy in CUMS rats. The expression of two critical markers in the process of autophagy, Beclin 1 and LC3 II/LC3 I were determined by western blotting 
A
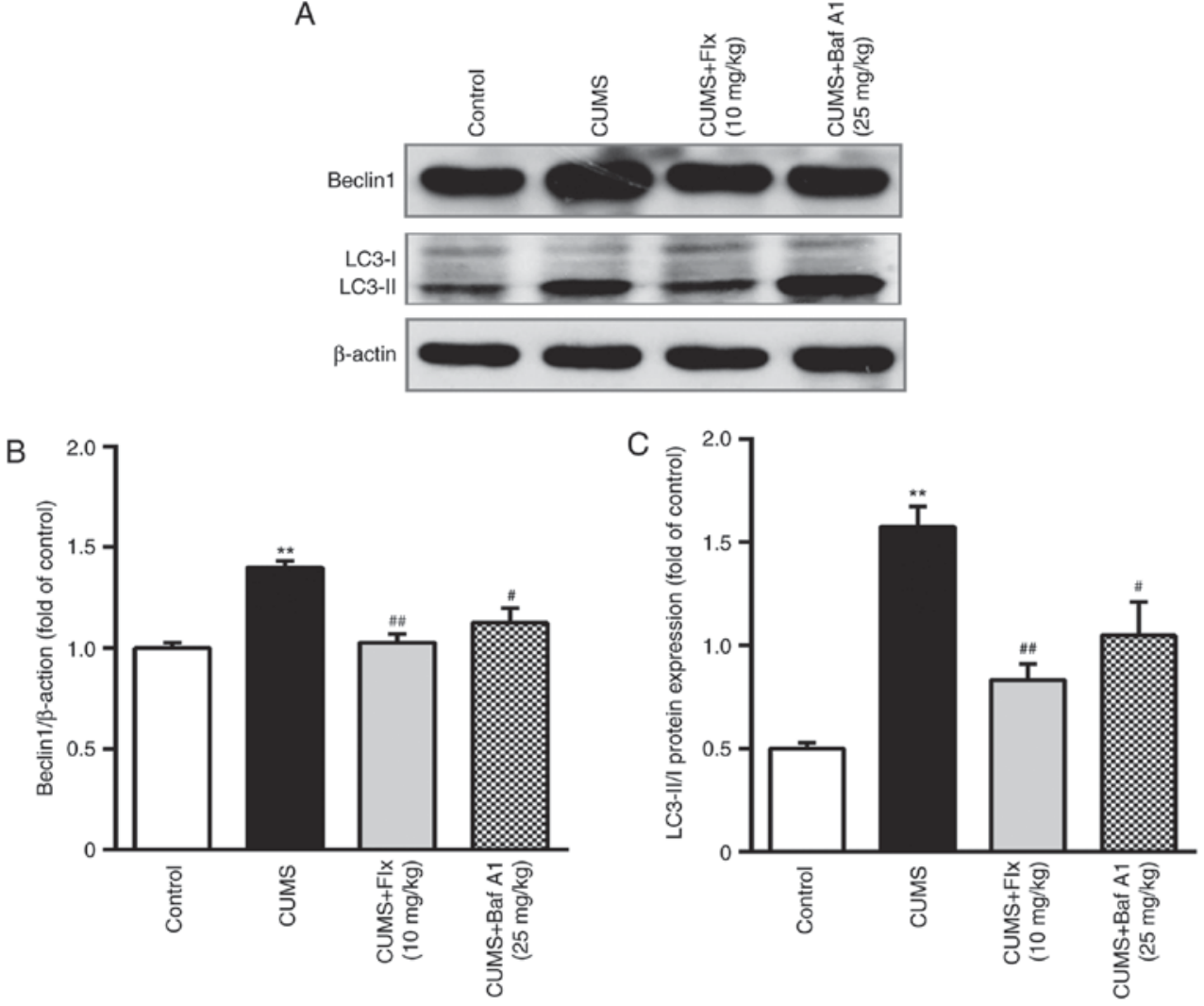

Figure 5. Effects of Baf A1 (25 mg/kg) or Flx (10 mg/kg) on CUMS-induced rat autophagy-associated proteins Beclin 1 and LC3-I/LC3-II. (A) Western blotting results; quantitative analysis of (B) Beclin-1 and (C) LC3-I/LC3-II expression. Data are expressed as the mean \pm standard error of the mean. $\mathrm{n}=10$ per group. ${ }^{* *} \mathrm{P}<0.01$ vs. Con; ${ }^{\#} \mathrm{P}<0.05,{ }^{\# \#} \mathrm{P}<0.01$ vs. CUMS. CUMS, chronic unpredictable mild stress; Flx, fluoxetine; Baf A1, Bafilomycin A1; LC3, microtubule-associated proteins $1 \mathrm{~A} / 1 \mathrm{~B}$ light chain 3.

(Fig. 5). The results demonstrated that the expression levels of Beclin 1 and LC3 II/LC3 I were significantly increased in the CUMS group. These alterations suggested that CUMS induced autophagic stress. However, the CUMS-exposed rats treated with Baf A1 $(25 \mathrm{mg} / \mathrm{kg})$ and fluoxetine $(10 \mathrm{mg} / \mathrm{kg})$ for 4 weeks exhibited significantly decreased Beclin1 expression and LC3 II/LC3 I ratios. These results suggested that Baf A1 inhibited autophagy in CUMS rats.

\section{Discussion}

In the present study, the results indicated that Baf A1 may possess antidepressant-like effects in the CUMS rat model. CUMS is considered to be one of the best animal models for mimicking the symptoms of depression and is a widely used model of depression (21). According to the observations from the present study, rats exposed to CUMS exhibited a significantly decreased body weight and percentage of sucrose preference, increased immobility time in the FST and decreased crossing time in the OFT. Administration of Baf A1 for 4 weeks significantly ameliorated the depressive-like behaviors in CUMS rats.

SYP and PSD 95 are associated with the survival and growth of neurons by regulating synaptic transmission and promoting synapse stability (22). These two synapse-associated proteins have been confirmed to serve important roles in neurogenesis and synaptogenesis (23). Clinical studies have indicated that the levels of PSD 95 and SYP are significantly decreased in patients with major depressive disorder $(24,25)$. A previous study reported that restraint stress tended to decrease the expression of SYP in the rat hippocampus, although this was restored to pre-stress levels after 2 weeks of treatment with venlafaxine (2). The present results revealed that CUMS caused a significant reduction in PSD 95 and SYP expression in the hippocampus, which was consistent with a previous study (26). Administration of Baf A1 for 4 weeks significantly increased the expression levels of SYP and PSD 95 in the CUMS group. The expression levels of synaptic plasticity proteins in the hippocampus were almost parallel to the antidepressant-like behavioral responses, as demonstrated by results obtained from western blotting and immunofluorescence analysis.

As mentioned, the activation of the inflammatory response system may serve an important role in the pathophysiology of depressive disorders (27). A previous study indicated that pro-inflammatory cytokines significantly increase in depressed patients and CUMS mice (28). In addition, antidepressants, including ketamine and fluoxetine, result in a decrease in pro-inflammatory cytokines, including IL-6 and TNF- $\alpha$ in the hippocampi of CUMS rats (29). The present results demonstrated that treatment with Baf A1 for 4 weeks significantly decreased the levels of IL- $1 \beta$ and TNF- $\alpha$ compared with the CUMS group, suggesting that Baf A1 inhibited inflammatory responses. In addition, high levels of pro-inflammatory cytokines may lead to a widespread increase in damaged proteins and autophagy (29). 
Autophagy is a ubiquitous phenomenon in eukaryotic cells that serves a key role in maintaining cellular homeostasis (30). Dysregulation of autophagy may cause a cellular 'traffic jam' during neuronal development and synaptic plasticity, leading to neurodevelopmental disorders (31). Previous research has suggested that autophagy is widely involved in the neural mechanism of depression, which may be a novel target for antidepressants (32). Rapamycin, a serine/threonine-protein kinase mTOR inhibitor, has also been reported to have antidepressant activity in animal models (33). In addition, autophagy markers, including Beclin 1 , are increased in the mouse brain following treatment with antidepressants (34). Autophagy may have favorable and unfavorable consequences in MDD, which may be the reason why certain patients with MDD remain resistant to certain antidepressants (31). The present study elucidated that Baf $\mathrm{A} 1$ reduced the expression of Beclin 1 and the ratio of LC3II/I in CUMS rats, which was consistent with results from previous studies $(12,15)$. These results indicated that Baf A1 alleviates depression-like behaviors, and this may be mediated by inhibition of autophagy.

In conclusion, the present study demonstrated that Baf A1 alleviates depression-like behaviors induced by CUMS, which may be mediated by inhibition of hippocampal inflammation, apoptosis and autophagy. It may therefore be proposed that Baf A1 may be a novel antidepressant candidate for the treatment of depression.

\section{Acknowledgements}

Not applicable.

\section{Funding}

The present study was supported by the Experimental Animal Science and Technology Plan Projects of Zhejiang Province (grant nos. 2018C37092, 2018 and 2014C37019, 2014]; Scientific and Technological Projects of Jiaxing (grant no. 2015AY23066, 2015); and College Students' Science and Technology Innovation Activity Plan and Talent Plan Project of Zhejiang Province (grant no. 2015R417004, 2015).

\section{Availability of data and materials}

The analyzed data sets generated during the present study are available from the corresponding author on reasonable request.

\section{Authors' contributions}

ZW wrote the manuscript and interpreted the data. SL and WP analyzed the data and revised the manuscript, YG searched the literature and collected the data. ZS designed the study.

\section{Ethics approval and consent to participate}

All animal experiments were approved by the Ethics Committee of the Medical College of Jiaxing University (Jiaxing, China; approval number JUMC2018-012) and all procedures adhered to the National Institutes of Health Guide for the Care and Use of Laboratory Animals.

\section{Patient consent for publication}

Not applicable.

\section{Competing interests}

The authors declare that they have no competing interests.

\section{References}

1. Barcelos-Ferreira R, Nakano EY, Steffens DC and Bottino CM: Quality of life and physical activity associated to lower prevalence of depression in community-dwelling elderly subjects from Sao Paulo. J Affect Disord 150: 616-622, 2013.

2. O'Neil A, Fisher AJ, Kibbey KJ, Jacka FN, Kotowicz MA, Williams LJ, Stuart AL, Berk M, Lewandowski PA, Taylor CB and Pasco JA: Depression is a risk factor for incident coronary heart disease in women: An 18-year longitudinal study. J Affect Disord 196: 117-124, 2016.

3. Chandrasekhar Y, Ramya EM, Navya K, Phani Kumar G and Anilakumar KR: Antidepressant like effects of hydrolysable tannins of Terminalia catappa leaf extract via modulation of hippocampal plasticity and regulation of monoamine neurotransmitters subjected to chronic mild stress (CMS). Biomed Pharmacother 86: 414-425, 2017.

4. Murphy JA and Byrne GJ: Prevalence and correlates of the proposed DSM-5 diagnosis of Chronic Depressive Disorder. J Affect Disord 139: 172-180, 2012.

5. Gaspersz R, Nawijn L, Lamers F and Penninx BWJH: Patients with anxious depression: Overview of prevalence, pathophysiology and impact on course and treatment outcome. Curr Opin Psychiatry 31: 17-25, 2018.

6. Kraus C, Castren E, Kasper S and Lanzenberger R: Serotonin and neuroplasticity-Links between molecular, functional and structural pathophysiology in depression. Neurosci Biobehav Rev 77: 317-326, 2017.

7. Kwon SE and Chapman ER: Synaptophysin regulates the kinetics of synaptic vesicle endocytosis in central neurons. Neuron 70: 847-854, 2011.

8. Politi P, Brondino N and Emanuele E: Increased proapoptotic serum activity in patients with chronic mood disorders. Arch Med Res 39: 242-245, 2008.

9. Komatsu M, Waguri S, Chiba T, Murata S, Iwata J, Tanida I, Ueno T, Koike M, Uchiyama Y, Kominami E and Tanaka K: Loss of autophagy in the central nervous system causes neurodegeneration in mice. Nature 441: 880-884, 2006.

10. Zschocke J, Zimmermann N, Berning B, Ganal V, Holsboer F and Rein T: Antidepressant drugs diversely affect autophagy pathways in astrocytes and neurons-dissociation from cholesterol homeostasis. Neuropsychopharmacology 36: 1754-1768, 2011.

11. Williamson LC and Neale EA: Bafilomycin A1 inhibits the action of tetanus toxin in spinal cord neurons in cell culture. J Neurochem 63: 2342-2345, 1994.

12. Pivtoraiko VN, Harrington AJ, Mader BJ, Luker AM, Caldwell GA, Caldwell KA, Roth KA and Shacka JJ: Low-dose bafilomycin attenuates neuronal cell death associated with autophagy-lysosome pathway dysfunction. J Neurochem 114: 1193-1204, 2010

13. Yuan N, Song L, Zhang S, Lin W, Cao Y, Xu F, Fang Y, Wang Z, Zhang H, Li X, et al: Bafilomycin A1 targets both autophagy and apoptosis pathways in pediatric B-cell acute lymphoblastic leukemia. Haematologica 100: 345-356, 2015.

14. Redmann M, Benavides GA, Berryhill TF, Wani WY, Ouyang X, Johnson MS, Ravi S, Barnes S, Darley-Usmar VM and Zhang J: Inhibition of autophagy with bafilomycin and chloroquine decreases mitochondrial quality and bioenergetic function in primary neurons. Redox Biol 11: 73-81, 2017.

15. Cui D, Wang L, Qi A, Zhou Q, Zhang X and Jiang W: Propofol prevents autophagic cell death following oxygen and glucose deprivation in PC12 cells and cerebral ischemia-reperfusion injury in rats. PLoS One 7: e35324, 2012. 
16. Qiu ZK, Zhong DS, He JL, Liu X, Chen JS and Nie H: The anxiolytic-like effects of puerarin are associated with the changes of monoaminergic neurotransmitters and biosynthesis of allopregnanolone in the brain. Metab Brain Dis 33: 167-175, 2018.

17. Li H, Lin S, Qin T, Li H, Ma Z and Ma S: Senegenin exerts anti-depression effect in mice induced by chronic un-predictable mild stress via inhibition of NF- $\kappa \mathrm{B}$ regulating NLRP3 signal pathway. Int Immunopharmacol 53: 24-32, 2017.

18. Porsolt RD, Bertin A and Jalfre M: Behavioral despair in mice: A primary screening test for antidepressants. Arch Int Pharmacodyn Ther 229: 327-336, 1977.

19. Livak KJ and Schmittgen TD: Analysis of relative gene expression data using real-time quantitative PCR and the 2(-Delta Delta C(T)) method. Methods 25: 402-408, 2001.

20. Zhao Z, Zhang L, Guo XD, Cao LL, Xue TF, Zhao XJ Yang DD, Yang J, Ji J, Huang JY and Sun XL: Rosiglitazone exerts an anti-depressive effect in unpredictable chronic mild-stress-induced depressive mice by maintaining essential neuron autophagy and inhibiting excessive astrocytic apoptosis Front Mol Neurosci 10: 293, 2017.

21. Yang JL, Liu X, Jiang H, Pan F, Ho CS and Ho RC: The effects of high-fat-diet combined with chronic unpredictable mild stress on depression-like behavior and Leptin/LepRb in male rats. Sci Rep 6: 35239, 2016.

22. Aguilar-Arredondo A, López-Hernández F, García-Velázquez L, Arias $\mathrm{C}$ and Zepeda A: Behavior-associated neuronal activation after kainic acid-induced hippocampal neurotoxicity is modulated in time. Anat Rec (Hoboken) 300: 425-432, 2017.

23. Duman $\mathrm{CH}$ and Duman RS: Spine synapse remodeling in the pathophysiology and treatment of depression. Neurosci Lett 601 20-29, 2015.

24. Zhao J, Bao AM, Qi XR, Kamphuis W, Luchetti S, Lou JS and Swaab DF: Gene expression of GABA and glutamate pathway markers in the prefrontal cortex of non-suicidal elderly depressed patients. J Affect Disord 138: 494-502, 2012

25. Gilabert-Juan J, Varea E, Guirado R, Blasco-Ibáñez JM, Crespo C and Nácher J: Alterations in the expression of PSA-NCAM and synaptic proteins in the dorsolateral prefrontal cortex of psychiatric disorder patients. Neurosci Lett 530: 97-102, 2012.
26. Liu XL, Luo L, Mu RH, Liu BB, Geng D, Liu Q and Yi LT: Fluoxetine regulates mTOR signalling in a region-dependent manner in depression-like mice. Sci Rep 5: 16024, 2015.

27. Leighton SP, Nerurkar L, Krishnadas R, Johnman C, Graham GJ and Cavanagh J: Chemokines in depression in health and in inflammatory illness: A systematic review and meta-analysis. Mol Psychiatry 23: 48-58, 2018.

28. Kochar B, Barnes EL, Long MD, Cushing KC, Galanko J, Martin CF, Raffals LE and Sandler RS: Depression is associated with more aggressive inflammatory bowel disease. Am J Gastroenterol 113: 80-85, 2018

29. Wang N, Yu HY, Shen XF, Gao ZQ, Yang C, Yang JJ and Zhang GF: The rapid antidepressant effect of ketamine in rats is associated with down-regulation of pro-inflammatory cytokines in the hippocampus. Ups J Med Sci 120: 241-248, 2015.

30. Du D, Hu L, Wu J, Wu Q, Cheng W, Guo Y, Guan R, Wang Y, Chen X, Yan X, et al: Neuroinflammation contributes to autophagy flux blockage in the neurons of rostral ventrolateral medulla in stress-induced hypertension rats. J Neuroinflammation 14: 169, 2017.

31. Jia J and Le W: Molecular network of neuronal autophagy in the pathophysiology and treatment of depression. Neurosci Bull 31: 427-434, 2015.

32. Ma J, Hou LN, Rong ZX, Liang P, Fang C, Li HF, Qi H and Chen HZ: Antidepressant desipramine leads to C6 glioma cell autophagy: Implication for the adjuvant therapy of cancer. Anticancer Agents Med Chem 13: 254-260, 2013.

33. Cleary C, Linde JA, Hiscock KM, Hadas I, Belmaker RH, Agam G, Flaisher-Grinberg S and Einat H: Antidepressive-like effects of rapamycin in animal models: Implications for mTOR inhibition as a new target for treatment of affective disorders. Brain Res Bull 76: 469-473, 2008.

34. Gassen NC, Hartmann J, Zschocke J, Stepan J, Hafner K, Zellner A, Kirmeier T, Kollmannsberger L, Wagner KV, Dedic N, et al: Association of FKBP51 with priming of autophagy pathways and mediation of antidepressant treatment response: Evidence in cells, mice, and humans. PLoS Med 11: e1001755, 2014. 